\title{
Probability Estimates for Continuous Spin Systems
}

D. Ruelle

Institut des Hautes Etudes Scientifiques, F-91440 Bures-sur-Yvette, France

\begin{abstract}
Probability estimates for classical systems of particles with superstable interactions [1] are extended to continuous spin systems.
\end{abstract}

\section{Notation and Assumptions}

On a lattice $\mathbb{Z}^{v}$ we consider continuous $d$-dimensional spins. A spin configuration in $\Lambda \subset \mathbb{Z}^{v}$ is thus a function $s_{\Lambda}: \Lambda \mapsto \mathbb{R}^{d}$; its value at $x \in \Lambda$ will be denoted by $s_{x}$.

If $x=\left(x^{1}, \ldots, x^{v}\right) \in \mathbb{Z}^{v}$, we write $|x|=\max _{i}\left|x^{i}\right|$. If $s=\left(s^{1}, \ldots, s^{d}\right) \in \mathbb{R}^{d}$, we write $|s|=\left(\sum_{i}\left(s^{i}\right)^{2}\right)^{1 / 2}=\sqrt{s^{2}}$.

A measure $\mu \geqq 0$ on $\mathbb{R}^{d}$ is given such that

$$
\int \mu(d s) e^{-\alpha s^{2}}<+\infty
$$

if $\alpha>0$, and $\mu$ is not identically 0 .

We shall call interaction a real function $U$ on all configurations in all finite $\Lambda \subset \mathbb{Z}^{v}$ satisfying the following conditions.

(a) $U$ is $\otimes^{\Lambda} \mu$-measurable on $\left(\mathbb{R}^{d}\right)^{\Lambda}$ and invariant under translations of $\mathbb{Z}^{v}$.

(b) Superstability. There exist $A>0, C \in \mathbb{R}$ such that if $s_{\Lambda} \in\left(\mathbb{R}^{d}\right)^{\Lambda}$ is a configuration on any finite $\Lambda$, then

$$
U\left(S_{\Lambda}\right) \geqq \sum_{x \in \Lambda}\left[A s_{x}^{2}-C\right]
$$

(c) Regularity. There exists a decreasing positive function $\Psi$ on the natural integers such that

$$
\sum_{x \in \mathbb{Z}^{v}} \Psi(|x|)<+\infty
$$

Furthermore if $\Lambda_{1}, \Lambda_{2}$ are disjoint finite subsets of $\mathbb{Z}^{v}$ and $s_{\Lambda_{1}}, s_{\Lambda_{2}}$ the restrictions to $\Lambda_{1}, \Lambda_{2}$ of a configuration $s_{\Lambda_{1} \cup A_{2}}$ on $\Lambda_{1} \cup \Lambda_{2}$, then

$$
\left|W\left(s_{\Lambda_{1} \cup \Lambda_{2}}\right)\right| \leqq \sum_{x \in \Lambda_{1}} \sum_{y \in \Lambda_{2}} \Psi(|y-x|) \frac{1}{2}\left(s_{x}^{2}+s_{y}^{2}\right)
$$


where we have written

$$
U\left(s_{\Lambda_{1} \cup \Lambda_{2}}\right)=U\left(s_{\Lambda_{1}}\right)+U\left(s_{\Lambda_{2}}\right)+W\left(s_{\Lambda_{1}}, s_{\Lambda_{2}}\right) .
$$

Condition (c) implies the following

(d) There are $r>0$ and $\lambda>0$ such that for all finite $\Lambda \subset \mathbb{Z}^{v}$

$$
\int_{\Sigma^{\Lambda}}\left(\prod_{x \in \Lambda} \mu\left(d s_{x}\right)\right) \exp \left[-U\left(s_{\Lambda}\right)\right]>\lambda^{-\operatorname{card} \Lambda}
$$

where $\Sigma=\left\{s \in \mathbb{R}^{d}:|s| \leqq r\right\}$. This is because, using (c), we have

$$
U\left(s_{\Lambda}\right) \leqq \sum_{x \in \Lambda} U\left(s_{x}\right)+\left(\sum_{x \in \Lambda} s_{x}^{2}\right) \sum_{y} \Psi(|y|)
$$

and, for sufficiently large $r, \int_{|s| \leqq r} \mu(d s)>0$.

Notice also that if there are $\varepsilon>0, B \in \mathbb{R}$ such that

$$
U\left(s_{\Lambda}\right) \geqq \sum_{x \in A}\left[(A+\varepsilon) s_{x}^{2}-B\left|s_{x}\right|\right]
$$

then (b) holds with $C=B / 4 \varepsilon$.

\section{Probability Estimates}

Let $\Delta \subset \Lambda \subset \mathbb{Z}^{v}, \Lambda$ finite. We denote by $s_{\Delta}$ the restriction to $\Delta$ of a configuration $s_{\Lambda}$ on $\Lambda$, and write

$$
\varrho_{\Delta}^{(\Lambda)}\left(s_{\Delta}\right)=Z_{\Lambda}^{-1} \int\left(\prod_{x \in \Lambda / \Delta} \mu\left(d s_{x}\right)\right) \exp \left[-U\left(s_{\Lambda}\right)\right]
$$

where

$$
Z_{\Lambda}=\int\left(\prod_{x \in \Lambda} \mu\left(d s_{x}\right)\right) \exp \left[-U\left(s_{\Lambda}\right)\right] .
$$

The probability estimates of this section are bounds on $\varrho_{\Delta}^{(\Lambda)}$, given in Theorem 2.2. below. To obtain these bound we imitate the arguments of [1]. That paper in effect treats a special case of the problem considered here, where $d=1$ and $\mu$ is carried by the natural integers. In [1], the probability estimates are obtained on the basis of technical results, which carry over immediately to the present case if the variable $n$ is allowed to vary in $\mathbb{R}^{d}$ rather than take natural integer values. As an example we transcribe below (Proposition 2.1) the main technical estimate of [1].

Given $\alpha>0$, we can choose an integer $P_{0}>0$ and for each $j \geqq P_{0}$ an integer $l_{j}>0$ such that

$$
\left|l_{j+1} / l_{j}-(1+2 \alpha)\right|<\alpha .
$$

We use the notation

$$
[j]=\left\{x \in \mathbb{Z}^{v}:|x| \leqq l_{j}\right\}, \quad V_{j}=\left(2 l_{j}+1\right)^{v}
$$


2.1. Proposition. Let $\varepsilon>0$ and $C \geqq 0$ be given, and let $\Psi$ be a decreasing positive function on the natural integers such that

$$
\sum_{x \in \mathbb{Z}^{v}} \Psi(|x|)<+\infty .
$$

If $\alpha$ is sufficiently small one can choose an increasing sequence $\left(\psi_{j}\right)$ such that $\psi_{j} \geqq 1$, $\psi_{j} \rightarrow \infty$, and fix $P>P_{0}$ so that the following is true.

Let $n(\cdot)$ be a function from $\mathbb{Z}$ to the reals $\geqq 0$. Suppose that there exists $q$ such that $q \geqq P$ and $q$ is the largest integer for which

$$
\sum_{x \in[q]} n(x)^{2} \geqq \psi_{q} V_{q}
$$

Then

$$
\sum_{x \in[q+1]} C+\sum_{x \in[q+1]} \sum_{y \notin[q+1]} \Psi(|y-x|) \frac{1}{2}\left(n(x)^{2}+n(y)^{2}\right) \leqq \varepsilon \sum_{x \in[q+1]} n(x)^{2} .
$$

This differs from Proposition 2.1 of [1] mostly by the fact that $n(\cdot)$ has real rather than integer values. Lemmas 2.2, 2.3, 2.4, and Proposition 2.5 of [1] similarly carry over to the present case.

To adapt Proposition 2.6 of [1] to $\varrho_{\Delta}^{(\Lambda)}$ some care is needed because we do not have in general $\varrho(\{0\})>0$. Since however we have (d) and the regularity condition (c) (rather than only lower regularity in [1]), we can write $\varrho_{\Delta}^{(\Lambda)}\left(s_{\Delta}\right)=\varrho^{\prime}+\varrho^{\prime \prime}$ where (3.30) and (3.31) of [1] are replaced (see Appendix) by

$$
\begin{aligned}
& \left.\varrho^{\prime} \leqq C^{\prime} \exp \left[\sum_{y \in \mathbb{Z}^{v}} \Psi(|y|)-A\right) s_{x}^{2}\right] \cdot \varrho_{\Delta \backslash\{x\}}^{(A)}\left(s_{\Delta \backslash\{x\}}\right) \\
& \varrho^{\prime \prime} \leqq \sum_{q \geqq P} e^{-C^{\prime \prime} \psi_{q+1} V_{q+1}+D^{\prime \prime} V_{q+1}} \cdot \exp \sum_{x \in[q+1] \cap \Lambda}\left[-(A-3 \varepsilon) s_{x}^{2}\right] \cdot \varrho_{\Delta \backslash[q+1]}^{(A)}\left(s_{\Delta \backslash[q+1]}\right)
\end{aligned}
$$

with some constants $C^{\prime}, C^{\prime \prime}, D^{\prime \prime}$. Therefore, by induction on card $\Delta$,

$$
\varrho_{\Delta}^{(\Lambda)}\left(s_{\Delta}\right) \leqq \exp \sum_{x \in \Delta}\left(E s_{x}^{2}+F\right)
$$

with some constants $E, F$.

We show now, following Proposition 2.7 of [1], that for any $\varepsilon>0$ one can choose $\delta$ independent of $(\Lambda), \Delta, s_{\Delta}$ such that

$$
\varrho_{\Delta}^{(A)}\left(s_{\Delta}\right) \leqq \exp \sum_{x \in \Delta}\left[-(A-3 \varepsilon) s_{x}^{2}+\delta\right] .
$$

We may assume $A>3 \varepsilon$. Let $\delta=(E+A-3 \varepsilon) \psi_{P} V_{P}+F$. If $\left|s_{x}\right| \leqq\left(\psi_{P} V_{P}\right)^{1 / 2}$ for each $x \in \Delta$, then (5) follows from (4). If $\left|s_{x}\right|>\left(\psi_{P} V_{P}\right)^{1 / 2}$ for some $x$, we put $x$ at the origin by a translation. Then $\varrho^{\prime}=0$, and $\varrho_{\Delta}^{(\Lambda)}\left(s_{\Delta}\right)=\varrho^{\prime \prime}$ so that, using (3) and induction,

$$
\begin{aligned}
& \varrho_{\Delta}^{(A)}\left(s_{\Delta}\right) \leqq \exp \sum_{x \in \Delta}\left[-(A-3 \varepsilon) s_{x}^{2}\right] \\
& \sum_{q \geqq P} e^{-C^{\prime \prime} \psi_{q+1} V_{q+1}+D V_{q+1}} e^{\delta \operatorname{card}(\Delta \backslash[q+1])} \\
& \leqq \exp \sum_{x \in \Delta}\left[-(A-3 \varepsilon) s_{x}^{2}\right] \cdot e^{\delta \operatorname{card}(\Delta \backslash[q+1])+F}
\end{aligned}
$$

and (4) follows. We have proved the following 
2.2. Theorem. Let $\varrho_{\Delta}^{(\Lambda)}\left(s_{\Delta}\right)$ be defined by (1) for an interaction $U$ satisfying (a), (b), (c). Given $A^{*}<A$, there exists $\delta$ independent of $\Lambda, \Delta, s_{\Delta}$ such that

$$
\varrho_{\Delta}^{(\Lambda)}\left(s_{\Delta}\right) \leqq \exp \sum_{x \in \Delta}\left[-A^{*} s_{x}^{2}+\delta\right] .
$$

2.3. Corollary. Let $\gamma \geqq 2$, and suppose that the superstability condition is strengthened to

$$
U\left(s_{\Lambda}\right) \geqq \sum_{x \in \Lambda}\left[A\left|s_{x}\right|^{\gamma}-C\right] .
$$

Then the conclusion of Theorem 2.2 can be strengthened to

$$
\varrho_{\Delta}^{(\Lambda)}\left(s_{\Delta}\right) \leqq \exp \sum_{x \in \Delta}\left[-A^{*}\left|s_{x}\right|^{\gamma}+\delta\right]
$$

Define $F: \mathbb{R}^{d} \mapsto \mathbb{R}^{d}$ by

$$
F S=\left\{\begin{array}{l}
s \quad \text { if } \quad|s| \leqq 1 \\
\left(|s|^{2 / \gamma-1}\right) s \text { if }|s| \geqq 1
\end{array}\right.
$$

and write $F\left(s_{x}\right)_{x \in \Lambda}=\left(F s_{x}\right)_{x \in \Lambda}$.

Let $\tilde{\mu}$ be the image by $F$ of the measure $\mu$, and let $\tilde{U}\left(s_{\Lambda}\right)=U\left(F s_{\Lambda}\right)$. Then $\tilde{U}$ is an interaction satisfying the conditions of Section 1 with respect to the measure $\tilde{\mu}$. In particular

$$
\begin{aligned}
\tilde{U}\left(s_{\Lambda}\right) & =U\left(F s_{\Lambda}\right) \geqq \sum_{x \in \Lambda}\left[A\left|F s_{x}\right|^{\gamma}-C\right] \\
& \geqq \sum_{x \in A}\left[A s_{x}^{2}-A-C\right]
\end{aligned}
$$

and

Therefore

$$
\begin{aligned}
\left|\tilde{W}\left(s_{\Lambda_{1} \cup \Lambda_{2}}\right)\right| & \leqq \sum_{x \in \Lambda_{1}} \sum_{y \in \Lambda_{2}} \Psi(|y-x|) \frac{1}{2}\left(\left|F s_{x}\right|^{2}+\left|F s_{y}\right|^{2}\right) \\
& \leqq \sum_{x \in \Lambda_{1}} \sum_{y \in \Lambda_{2}} \Psi(|y-x|) \frac{1}{2}\left(s_{x}^{2}+s_{y}^{2}\right) .
\end{aligned}
$$

$$
\begin{aligned}
\varrho_{\Delta}^{(\Lambda)}\left(s_{\Delta}\right) & =\varrho_{\Delta}^{(\Lambda)}\left(F^{-1} s_{\Delta}\right) \leqq \exp \sum_{x \in \Delta}\left[-A^{*}\left|F^{-1} s_{x}\right|^{2}+\delta\right] \\
& \leqq \exp \sum_{x \in \Delta}\left[-A^{*}\left|s_{x}\right|^{\gamma}+\delta\right] .
\end{aligned}
$$

2.4. Corollary. Suppose that

$$
U\left(s_{\Lambda}\right)=\tilde{U}\left(s_{\Lambda}\right)+\sum_{x \in \Lambda} V\left(s_{x}\right)
$$

and that $\tilde{U}$ is an interaction satisfying the conditions of Section 1 with respect to the measure $\tilde{\mu}=e^{-v} \mu$. Then Theorem 2.2 can be replaced by

$$
\varrho_{\Delta}^{(\Lambda)}\left(s_{\Delta}\right) \leqq \exp \sum_{x \in \Delta}\left[-A^{*}\left|s_{x}\right|^{\gamma}+\delta-V\left(s_{x}\right) \mid .\right.
$$

This is because

$$
\varrho_{\Delta}^{(\Lambda)}\left(s_{\Delta}\right)=\exp \left[-\sum_{x \in \Delta} V\left(s_{x}\right)\right] \varrho_{\Delta}^{(\Lambda)}\left(s_{\Delta}\right)
$$

where $\varrho$ is defined by (1) with $\mu, U$ replaced by $\tilde{\mu}, \tilde{U}$. 


\section{Appendix}

We sketch here the proofs of (2) and (3), using notation which is either that of [1], or has obvious meaning.

Proof of (2).

$$
\begin{aligned}
\varrho^{\prime}= & Z_{\Lambda}^{-1} \int_{R} \mu^{\Lambda \backslash \Delta}\left(d s_{\Lambda \backslash \Delta}\right) \exp \left[-U\left(s_{x}\right)-U\left(s_{\Lambda \backslash\{x\}}\right)-W\left(s_{x}, s_{\Lambda \backslash\{x\}}\right)\right] \\
\leqq & e^{-U\left(s_{x}\right)} Z_{\Lambda}^{-1} \int_{R} \mu^{\Lambda \backslash \Delta}\left(d s_{\Lambda \backslash \Delta}\right) \exp \left[-U\left(s_{\Lambda \backslash\{x\}}\right)-W\left(s_{x}^{\prime}, s_{\Lambda \backslash\{x\}}\right)\right] \\
& \cdot \exp \left[\left(\frac{1}{2} \sum_{y} \Psi(|y|)\right)\left(s_{x}^{2}+s_{x}^{\prime 2}\right)+2 D^{\prime}\right] \\
\leqq & \lambda e^{2 D^{\prime}} \exp \left[-A s_{x}^{2}+C+\left(\frac{1}{2} \sum_{y} \Psi(|y|)\right) s_{x}^{2}\right] \\
& \cdot \sup _{s_{x}^{\prime} \in \Sigma} \exp \left[\left(\frac{1}{2} \sum_{y} \Psi(|y|)\right) s_{x}^{\prime 2}\right] \\
& \cdot Z_{\Lambda}^{-1} \int_{\Sigma} \mu\left(d s_{x}^{\prime}\right) \int_{R} \mu^{\Lambda \backslash \Delta}\left(d s_{\Lambda \backslash \Delta}\right) \exp \left[-U\left(s_{\Lambda}^{*}\right)\right] \\
\leqq & C^{\prime} \exp \left[\left(\sum_{y} \Psi(|y|)-A\right) s_{x}^{2}\right] \cdot \varrho_{\Delta \backslash\{x\}}^{(\Lambda)}\left(s_{\Delta \backslash\{x\}}\right) .
\end{aligned}
$$

Proof of (3).

$$
\begin{aligned}
& \varrho^{\prime \prime}=\sum_{q \geqq P} Z_{\Lambda}^{-1} \int_{R_{q}} \mu^{\Lambda \backslash \Delta}\left(d s_{\Lambda \mid A}\right) \exp \left(-U\left(s_{[q+1] \cap \Lambda}\right)\right) \\
& \cdot \exp \left(-W\left(s_{[q+1] \cap \Lambda}, s_{\Lambda \cap[q+1]}\right)\right) \exp \left(-U\left(s_{\Lambda \backslash[q+1]}\right)\right) \\
& \leqq \sum_{q \geqq P} Z_{\Lambda}^{-1} \int_{R_{q}} \mu^{\Lambda \backslash \Delta}\left(d s_{\Lambda \backslash \Delta}\right) \exp \sum_{x \in[q+1] \cap \Lambda}\left[-A s_{x}^{2}+C\right] \\
& \cdot \exp \sum_{x \in[q+1] \cap \Lambda} \sum_{y \in \Lambda[q+1]} \Psi(|y-x|) \frac{1}{2}\left(s_{x}^{2}+s_{y}^{2}\right) \\
& \cdot \exp \sum_{x \in[q+1] \cap \Lambda} \sum_{y \in \Lambda \backslash[q+1]} \Psi(|y-x|) \frac{1}{2}\left(s_{x}^{\prime 2}+s_{y}^{2}\right) \\
& \cdot \exp \left[-W\left(s_{[q+1] \cap \Lambda^{\prime}}^{\prime} S_{\Lambda \backslash[q+1]}\right)-U\left(s_{\Lambda \backslash[q+1]}\right)\right] \\
& \leqq \sum_{q \geqq P} Z_{\Lambda}^{-1} \int_{R_{q}} \mu^{\Lambda \backslash 4}\left(d s_{\Lambda \backslash 4}\right) \\
& \cdot \exp \left[-(A-3 \varepsilon) \sum_{x \in[q+1] \cap A} S_{x}^{2}-C^{\prime \prime} \Psi_{q+1} V_{q+1}\right] \\
& \cdot \exp \left(\frac{1}{2} \sum_{y} \Psi(|y|) \sum_{x \in[q+1] \cap A} S_{x}^{\prime 2}\right. \\
& \cdot \exp \left[-W\left(s_{[q+1] \cap A}^{\prime}, s_{\Lambda \backslash[q+1]}\right)-U\left(s_{\Lambda \backslash[q+1]}\right)\right] \\
& \leqq \sum_{q \geqq P} \exp \sum_{x \in[q+1] \cap \Delta}\left[-(A-3 \varepsilon) s_{x}^{2}\right] \\
& \cdot e^{-C^{\prime \prime}} \psi_{q+1} V_{q+1}\left[\int \mu(d s) e^{-(A-3 \varepsilon) s^{2}}\right]^{|[q+1] \cap \Lambda \backslash \Delta|} \\
& \cdot\left(\sup _{s^{\prime} \in \Sigma} \exp \left[\left(\frac{1}{2} \sum_{y} \Psi(|y|)\right) s^{\prime 2}\right]\right)^{|[q+1] \cap \Lambda|} \lambda^{|[q+1] \cap| \Delta \mid} \\
& \cdot Z_{\Lambda}^{-1} \int_{\Sigma^{[q+1] \cap \Lambda}} \mu^{[q+1] \cap \Lambda}\left(d s_{[q+1] \cap \Lambda}^{\prime}\right) \int \mu^{\Lambda \backslash[q+1]}\left(d s_{\Lambda \backslash[q+1] \backslash \Lambda}\right) e^{-U\left(s_{\Lambda}^{*}\right)} \\
& \leqq \sum_{q \geqq P} \exp \sum_{x \in[q+1] \cap A}\left[-(A-3 \varepsilon) S_{x}^{2}\right] \\
& \cdot e^{-C^{\prime \prime} \psi_{q+1} V_{q+1}+D^{\prime \prime} V_{q+1}} \varrho_{\Delta \backslash[q+1]}^{(\Lambda)}\left(S_{\Delta \backslash[q+1]}\right) .
\end{aligned}
$$


Acknowledgements. I am indebted to Joel Lebowitz for questions and discussions which originated the present note. Applications of the estimates in this note to the thermodynamic limit and related problems will be made in a separate paper by J.L. Lebowitz and E. Presutti.

\section{Reference}

1. Ruelle,D.: Superstable interactions in classical statistical mechanics. Commun. math. Phys. 18, $127-159$ (1970)

Communicated by G. Gallavotti

Received April 12, 1976 